\title{
Expression of cyclooxygenase-2 and vascular endothelial growth factor in primary central nervous system lymphomas
}

\author{
YASUO SUGITA $^{1}$, HIDEKI KOMATANI ${ }^{1,2}$, KOICHI OHSHIMA ${ }^{1}$, \\ MINORU SHIGEMORI ${ }^{2}$ and AKIHIKO NAKASHIMA ${ }^{3}$ \\ Departments of ${ }^{1}$ Pathology and ${ }^{2}$ Neurosurgery, Kurume University School of Medicine; \\ ${ }^{3}$ Department of Pathology, Fukuoka Saisekai General Hospital, Kurume, Fukuoka 830-0011, Japan
}

Received March 14, 2007; Accepted May 30, 2007

\begin{abstract}
Cyclooxygenase (COX) is the rate-limiting enzyme that catalyzes the initial step in biosynthesis of prostaglandins (PGs) from arachidonic acid. COX-2 has been associated with inflammatory processes and tumorigenesis. In order to investigate the correlation between VEGF, COX-2 expression, and tumorigenesis in primary central nervous system lymphomas (PCNSLs), the present study assessed 26 cases of PCNSL by immunostaining for VEGF and COX-2. Immunohistochemical studies were evaluated as follows: (-), no staining; (1+), 0-30\% positive cells; $(2+), 30-60 \%$ positive cells; $(3+),>60 \%$ positive cells. VEGF expression was detected in 21 of 26 cases; of these, 14, 1 and 6 were scored as 3+, 2+ and 1+, respectively. COX-2 expression was detected in 22 of 26 cases; of these, 14,4 and 4 were scored as $3+, 2+$ and $1+$, respectively. For double immunofluorescence, 20 of 26 cases that were detected with both VEGF and COX-2 were examined and almost all tumor cells coexpressed both VEGF and COX-2 in the examined cases. However, COX-2 and VEGF expression in PCNSLs did not correlate with neoangiogenesis and patient survival in the present study, in contrast to previous findings in systemic lymphomas. It is suggested that the high frequency of COX-2 and VEGF coexpression in PCNSLs may be associated with tumorigenesis of PCNSLs and could possibly lead to a future therapeutic trial of PCNSLs with selective COX-2 inhibitor therapy.
\end{abstract}

\section{Introduction}

The incidence of primary central nervous system lymphomas (PCNSLs) is quite low, currently accounting for 1-2\% of

Correspondence to: Dr Yasuo Sugita, Department of Pathology, Kurume University School of Medicine, Asahimachi 67, Kurume, Fukuoka 830-0011, Japan

E-mail: sugita_yasuo@med.kurume-u.ac.jp

Key words: cyclooxygenase 2, central nervous system lymphomas, angiogenesis, vascular endothelial growth factor lymphomas, but this incidence has been increasing steadily and the most recent analysis reveals that it now represents $4 \%$ of all brain tumors (1). An increasing incidence of PCNSLs has been seen not only in patients associated with acquired immunodeficiency syndrome (AIDS), but also in immunocompetent hosts $(1,2)$. The majority of PCNSLs are histopathologically confirmed as diffuse large B-cell lymphoma (DLBCL) $(2,6)$. However, distinct characteristics of PCNSLs have been demonstrated, including poor prognosis compared with non-PCNSLs (3-5). Hence, new molecular targets are needed for the prevention and treatment of PCNSLs.

Cyclooxygenase (COX) is the rate-limiting enzyme that catalyzes the initial step in biosynthesis of prostaglandins (PGs) from arachidonic acid. Two COX enzymes have been described; COX-1 is the constitutive form and is regarded as a housekeeping gene, whereas COX-2 has been associated with inflammatory processes and tumorigenesis (7-23). Recent evidence suggests that excessive COX-2 overexpression plays a role in tumor growth and spread also because of its ability to regulate tumor vascularization by modulating production of several angiogenic factors, including vascular endothelial growth factor (18-20,23). Moreover, it has been reported that inhibition of $\mathrm{COX}-2$ could be a new targeted therapy for B-cell lymphomas $(21,24)$. From this perspective, we studied a series of 26 PCNSLs to investigate the correlation between vascular endothelial growth factor (VEGF) and COX-2 expression and the pathogenesis of PCNSLs.

\section{Materials and methods}

Cases. Surgical tissue samples $(n=26)$ from 26 patients with PCNSL were studied. All tumor specimens were retrieved from the archives of Kurume University and their affiliated hospitals between 1989 and 2003. The clinical information for the patients was also retrieved from these archives.

Histological and immunohistochemical studies. Tissue samples were fixed in $10 \%$ buffered formalin, embedded in paraffin and processed conventionally for histology and immunohistochemistry. Sections $(5 \mu \mathrm{m})$ were stained using hematoxylin and eosin (HE) for histological evaluation. The remaining serial unstained sections were used for immuno- 
Table I. Summary of clinicopathological data.

\begin{tabular}{|c|c|c|c|c|c|c|}
\hline Case no. & Age/gender & Histopathology & VEGF & COX-2 & IMVD & Follow-up \\
\hline 1 & $59 / \mathrm{M}$ & DL, CBV & $3+$ & $3+$ & 11.6 & ANED 1 month \\
\hline 2 & $56 / \mathrm{F}$ & DL, CBV & $3+$ & $3+$ & 5.0 & DOD 20 months \\
\hline 3 & $56 / \mathrm{M}$ & $\mathrm{DL}, \mathrm{CBV}$ & & 0 & 30.3 & ANED 10 months \\
\hline 4 & $57 / \mathrm{M}$ & DL, IMBV & $1+$ & $2+$ & 5.0 & Unknown \\
\hline 5 & $83 / \mathrm{F}$ & DL, IMBV & 0 & $1+$ & 13.3 & ANED 12 months \\
\hline 6 & $83 / F$ & DL, CBV & $3+$ & $3+$ & 5.1 & ANED 17 months \\
\hline 7 & $62 / \mathrm{F}$ & DL, CBV & $3+$ & $2+$ & 5.3 & Unknown \\
\hline 8 & $68 / \mathrm{F}$ & DL, CBV & $1+$ & $1+$ & 5.1 & Unknown \\
\hline 9 & $61 / \mathrm{M}$ & DL, CBV & $3+$ & $3+$ & 5.0 & ANED 94 months \\
\hline 10 & $70 / \mathrm{M}$ & DL, CBV & 0 & 0 & 10.0 & DOD 20 months \\
\hline 11 & $48 / \mathrm{M}$ & $\mathrm{DL}, \mathrm{CBV}$ & $1+$ & $1+$ & 11.0 & ANED 60 months \\
\hline 12 & $54 / \mathrm{F}$ & DL, CBV & $1+$ & 0 & 20.0 & ANED 2 months \\
\hline 13 & $70 / \mathrm{F}$ & DL, CBV & 0 & $3+$ & 22.3 & ANED 1 month \\
\hline 14 & $71 / \mathrm{F}$ & DL, CBV & $1+$ & $3+$ & 31.0 & ANED 4 months \\
\hline 15 & 79/M & DL, CBV & $2+$ & $3+$ & 20.0 & DOD 5 months \\
\hline 16 & $67 / \mathrm{M}$ & DL, CBV & $3+$ & $1+$ & 10.0 & ANED 18 months \\
\hline 17 & $70 / \mathrm{F}$ & DL, CBV & $3+$ & $3+$ & 36.6 & DOD 6 months \\
\hline 18 & $59 / \mathrm{M}$ & DL, CBV & $3+$ & $3+$ & 16.3 & DOD 6 months \\
\hline 19 & $79 / \mathrm{F}$ & DL, CBV & 0 & 0 & 19.0 & DOD 1 month \\
\hline 20 & $70 / \mathrm{M}$ & DL, IMBV & $3+$ & $3+$ & 18.0 & DOD 1 month \\
\hline 21 & $56 / F$ & DL, CBV & $3+$ & $3+$ & 12.0 & ANED 4 months \\
\hline 22 & $66 / \mathrm{M}$ & DL, CBV & $3+$ & $3+$ & 13.0 & ANED 5 months \\
\hline 23 & $54 / \mathrm{M}$ & DL, CBV & $1+$ & $2+$ & 35.2 & DOD 4 months \\
\hline 24 & $60 / F$ & DL, CBV & $3+$ & $3+$ & 35.0 & DOD 10 months \\
\hline 25 & $62 / F$ & DL, IMBV & $3+$ & $3+$ & 15.2 & DOD 11 months \\
\hline 26 & $56 / \mathrm{M}$ & DL, CBV & $3+$ & $2+$ & 16.0 & DOD 14 months \\
\hline
\end{tabular}

ANED, alive and no evidence of disease; CBV, centroblastic variant; COX-2, cyclooxygenase-2; DL, diffuse large; DOD, died of disease; F, female; IMBV, immunoblastic variant; IMVD, intratumoral microvessel density; M, male; VEGF, vascular endothelial growth factor.

histochemistry. All specimens were histologically diagnosed according to the World Health Organization criteria for tumors of the central nervous system (6). Immunohistochemical studies were performed using peroxidase avidin-biotin methods (LASB kit, Dako Corp., Carpinteria, CA, USA) on paraffin sections following heat-induced antigen retrieval. Primary antibodies were directed toward CD20 (dilution 1:50; DakoCytomation, Glostrup, Denmark), CD79a (dilution 1:50; DakoCytomation), CD3 (dilution 1:50; DakoCytomation), CD45RO (dilution 1:100; DakoCytomation), Cyclooxygenase-2 (COX-2, 1:250; Cayman Chemical, MI, USA), vascular endothelial growth factor (VEGF, dilution 1:10; R\&D Systems, Minneapolis, USA), and endoglin (CD105, dilution 1:50; Novocastra, Newcastle, UK).

Immunohistochemical studies were evaluated as follows: $(-)$, no staining; $(1+), 0-30 \%$ positive cells; $(2+), 30-60 \%$ positive cells; $(3+),>60 \%$ positive cells.

Immunohistochemical evaluation was performed by two observers (Y.S. and H.K.) in independent readings. Cases that varied significantly between readers were re-evaluated in order to arrive at a consensus.
Quantitation of CD105 immunostained vessels. Endoglin (CD105) is predominantly expressed on cellular lineages within the vascular system and is overexpressed on proliferating endothelial cells that participate in tumor angiogenesis, with weak or negative expression in the vascular endothelium of normal tissues (25). Thus, we used endoglin (CD105) to evaluate neoangiogenesis of PCNSLs. After scanning the immunostained section at low magnification, three areas of tumor with the greatest number of distinctly highlighted areas (hot-spots) were counted on a x200 field. Any single cell or spot that was stained by the immunohistochemical marker was counted as a vessel. The average counts from the three areas were recorded as the CD105-IMVD for each tumor to measure the degree of angiogenesis.

Double immunofluorescence. The slides were deparaffinized and soaked in $0.01 \mathrm{M}$ citrate buffer $\left(\mathrm{pH} 6.0\right.$ ) at $90^{\circ} \mathrm{C}$ for $40 \mathrm{~min}$ for antigen retrieval. The samples were treated with $10 \mathrm{mg} /$ $\mathrm{ml}$ bovine serum albumin (BSA) to inhibit non-specific antibody binding and were then incubated with the primary murine antibody VEGF for $1 \mathrm{~h}$ at $37^{\circ} \mathrm{C}$. After washing thrice with 


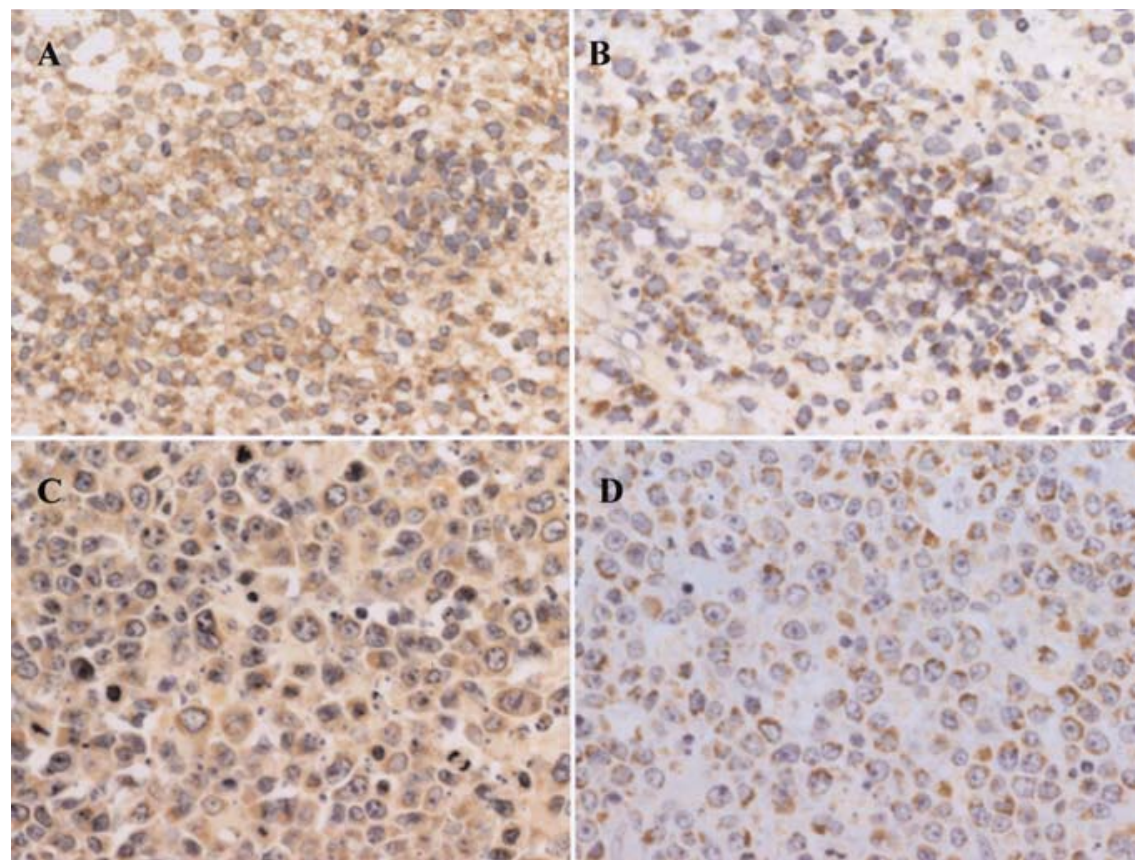

Figure 1. Illustration of immunohistochemical findings for VEGF and COX-2 proteins in primary central nervous system lymphoma. (A) VEGF proteinpositive lymphoma showing cytoplasmic staining (3+) (case 22). (B) COX-2 protein-positive lymphoma showing cytoplasmic staining (3+) (case 22). (C) VEGF protein-positive lymphoma showing cytoplasmic staining (3+) (case 1). (D) COX-2 protein-positive lymphoma showing cytoplasmic staining (3+) (case 1).

PBS ( $\mathrm{pH} 7.2$ ), the samples were incubated with fluoresceinisothiocyanate (FITC)-labeled secondary rabbit polyclonal antibody against murine immunoglobulin $\mathrm{G}$ ( $\mathrm{IgG}$ ) for $30 \mathrm{~min}$ at $37^{\circ} \mathrm{C}$. To inactivate the primary and secondary antibodies, the samples were heated in the citrate buffer at $90^{\circ} \mathrm{C}$ for $15 \mathrm{~min}$. This treatment made it possible to carry out the second immunoreaction with the monoclonal antibody. For the second immunoreaction, the same procedure was repeated; the samples were treated with $10 \mathrm{mg} / \mathrm{ml} \mathrm{BSA}$, incubated with another primary antibody $\mathrm{COX}-2$, and then incubated with rhodamine-labeled secondary antibody. After washing with PBS, FITC-labeled and rhodamine-labeled samples were examined using a fluorescence microscope (Olympus BX60, Tokyo, Japan). To detect non-specific antibody binding, control sections were incubated with either normal murine or rabbit serum or PBS instead of primary antibody. No staining was observed in these control samples.

In situ mRNA hybridization. Preparation of frozen tissues (case no. 22) and in situ hybridization were performed as described (26). The sequence of the oligonucleotide probe for VEGF is TGGTGATGTTGGACTCCTCAGTGGGC.

Statistical analysis. Statistical analyses were performed by using the Stat Mate III for Windows Version software package (ATMS, Tokyo, Japan). The survival rate was computed using the Kaplan-Meier method. Patients were censored on loss-to-follow-up at the time of analysis. Survival curves were computed between subgroups of patients using the Cox-Mantel test. Comparison of the tumor cell expression of COX-2 or CD105-IMVD were assessed by Pearson correlation.

\section{Results}

Clinical features. The clinical features of the 26 patients are summarized in Table I. Follow-up data are available in 23 of the 26 cases. The legal limitation regarding personal information prevented us from analyzing the follow-up data of some cases. Therefore, the survival curves were established based on the data from only 23 patients. Most patients received adjuvant radiation and chemotherapy, while 2 (cases 19 and 20) were not treated with either adjuvant radiation or chemotherapy due to their poor overall conditions. Eighteen patients (cases 1-18) received CHOP (cyclophosphamide, doxorubicin, vincristine, prednisone) chemotherapy, while 6 (cases 21-26) were treated by high doses of methotrexate.

Histological and immunohistochemical features of PCNSLs. The histological characteristics of the 26 PCNSLs are summarized in Table I. Immunohistochemically, all cases expressed CD20 or CD79a, but were negative for CD3 and CD45RO (data not shown). VEGF expression was detected in 21 of 26 cases; of these, 14, 1 and 6 were scored as 3+, 2+ and $1+$, respectively. COX-2 expression was detected in 22 of 26 cases; of these, 14, 4 and 4 were scored as 3+, 2+ and $1+$, respectively (Table I, Fig. 1). Eleven of 21 cases (52\%) that highly expressed VEGF (3+) also showed high level of COX-2 (3+). For double immunofluorescence, 20 of 26 cases that were detected with both VEGF and COX-2 were examined and almost all tumor cells coexpressed both VEGF and COX-2 in the examined cases (Fig. 2).

The average counts from the three areas were recorded as the CD105-IMVD for each tumor (Table I). To gain a better understanding of the correlation with VEGF, COX-2 

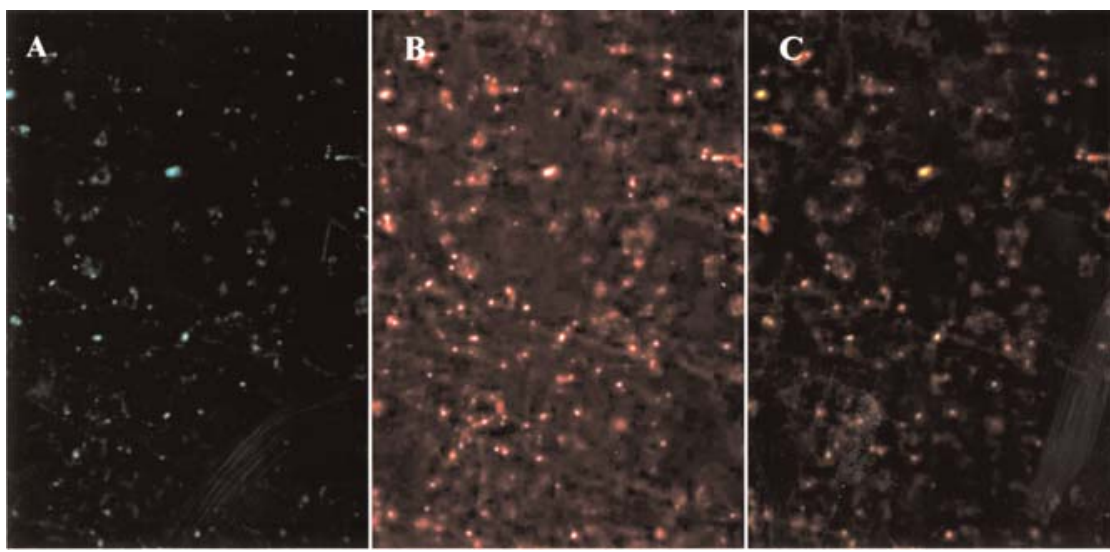

Figure 2. Representative illustration of double immunofluorescence findings for VEGF and COX-2 proteins in primary central nervous system lymphoma (case 22). (A) An intense cytoplasmic expression of VEGF is noted (green fluorescence image). (B) An intense cytoplasmic expression of COX-2 is noted (red fluorescence image). (C) Colocalization of VEGF and COX-2 in lymphoma cells. Their characteristics are demonstrated in the merged image.

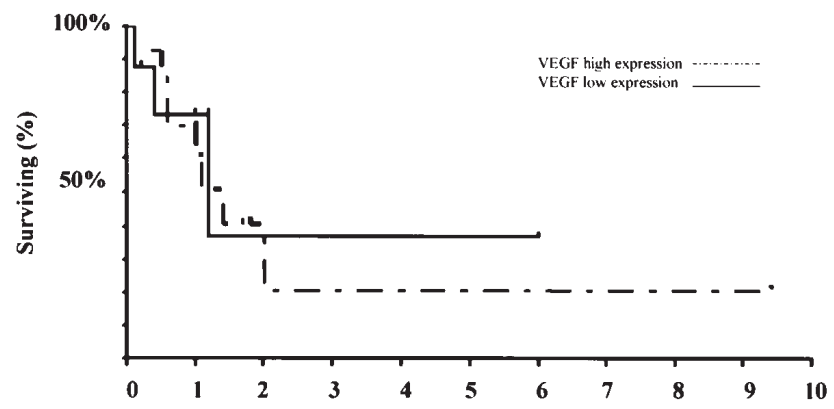

Figure 3. Survival curves (Kaplan-Meier) for patients with VEGF high expression $(\mathrm{n}=14)$ and VEGF low expression $(\mathrm{n}=7)$. There are no significant differences in the 5-year survival rate between these two groups $(\mathrm{p}<0.01$, Cox-Mantel test).

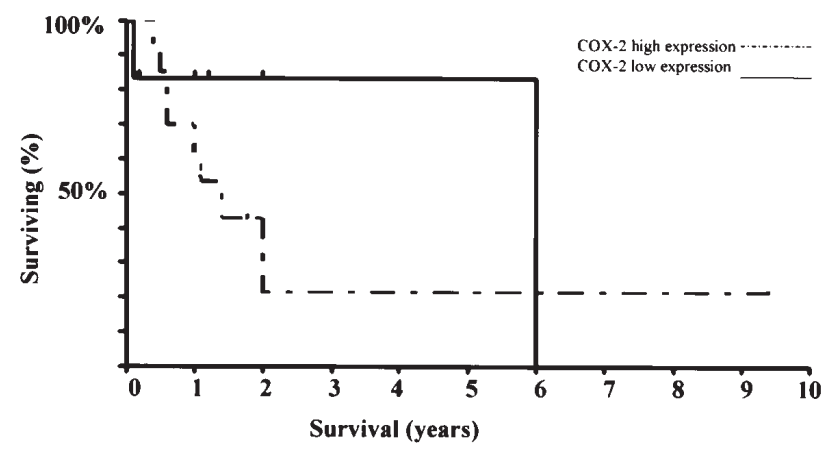

Figure 4. Survival curves (Kaplan-Meier) for patients with COX-2 high expression $(n=14)$ and COX-2 low expression $(n=8)$. There are no significant differences in the 5-year survival rate between these two groups $(\mathrm{p}=0.21$, Cox-Mantel test).

expression and the CD105-IMVD in PCNSLs, statistical analyses between each of the relevant groups were performed. Neither VEGF expression nor COX-2 correlated with the CD105-IMVD (VEGF: $\mathrm{r}=-0.19, \mathrm{p}=0.35, \mathrm{COX}-2$ : $\mathrm{r}=0.05, \mathrm{p}=0.79$ ).

According to the score of VEGF or COX-2, patients were classified into lower-score $(\leq 2)$ and higher-score patients $(>3)$. When VEGF was used as a marker, the survival rate

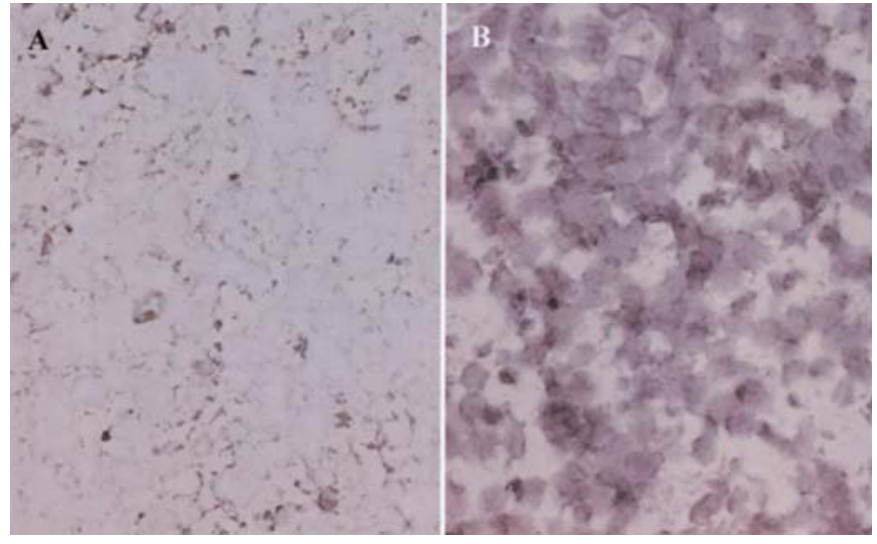

Figure 5. (A) The signals of VEGF expression are detected in the cytoplasm of tumor cells in situ mRNA hybridization (case 22). (B) Negative control of sense VEGF probe is shown (case 22).

between these two groups demonstrated no significant difference $(p=0.34)$ (Fig. 3). When COX-2 was used as a marker, the survival rate between these two groups demonstrated no significant difference ( $\mathrm{p}=0.21$ ) (Fig. 4).

In situ mRNA hybridization. The signals of VEGF expression were detected in the cytoplasm of tumor cells with in situ mRNA hybridization (Fig. 5).

\section{Discussion}

Numerous studies have revealed that COX-2 and VEGF play a critical role in the development of neoplasms in a variety of ways (7-23). For example, Gallo et al have reported that COX-2 activation induces VEGF production in human epidermoid cell lines, which is abolished by the COX inhibitors indomethacin and celecoxib, suggesting that the COX-2 pathway may play a central role in squamous cell carcinoma by modulating VEGF production (14). Fujiwaki et al also have observed upregulation of protein and mRNA expression for COX-2 and VEGF in endometrial cancers compared to normal endometrium (13). As such, they hypothesized that 
COX-2 regulates tumor-induced angiogenesis in endometrial cancer via VEGF. In brain tumors, Shono et al have reported that high COX-2 expression in tumor cells correlates with the increasing histological grade of astrocytic gliomas (12). Hara and Okayasu have described a stepwise increase in immunoreactive scores for COX-2 and VEGF from astrogliosis, through low- to high-grade astrocytoma (19). They also pointed out that the findings for COX-2 and VEGF-related angiogenesis raise the possibility that the COX-2 pathway may contribute to astrocytic tumorigenesis by promoting new vessel formation with prognostic implications. Recently, several studies have suggested that the growth of hematopoietic neoplasms also depends on angiogenesis $(21,27)$. Salven et al have demonstrated that simultaneous serum elevation of VEGF and basic fibroblast growth factor is an independent predictor of poor prognosis in non-Hodgkin's lymphoma (27). Hazar et al have investigated the role of COX-2 and VEGF in malignant lymphoma (28). They showed that prostaglandins regulate the VEGF expresson and that COX-2 activity is able to activate tumor angiogenesis. They also demonstrated COX-2 and VEGF might have a common induction pathway in malignant lymphoma. There is scarce data regarding tumor angiogenesis in PCNSLs. However, we have previously demonstrated that the growth of PCNSLs is dependent on angiogenesis. Particularly, the microvascular density determined by anti-endoglin (CD105) monoclonal antibody correlates with patient survival (25). In the present study, we show high frequency and colocalization of VEGF and COX-2 protein in tumor cells in PCNSLs. In addition, mRNA expression was observed for VEGF in one case. These findings suggested that VEGF and COX-2 contribute to the tumorigenesis in PCNSLs, similar to systemic lymphomas. Although most PCNSLs in immunocompetent patients are diffuse large B-cell lymphoma, they are characterized by poor prognosis compared with the same type of systemic lymphomas (3-5). Some investigators have speculated that the poor PCNSL prognosis may in part be due to biologic aggressiveness associated with its activated B cell-like pattern $(2,5)$. In the present study, COX-2 and VEGF expression in tumor cells did not correlate with microvascular density and patient survival, in contrast with the findings for systemic lymphomas. This discrepancy is presumably due, in part, to the small number of cases examined. In contrast, we have previously reported that PCNSLs do not require sufficient neoangiogenesis at the start of PCNSLs, also in contrast with systemic lymphomas (25). From this perspective, the reason for the lack of correlation between COX-2 and VEGF overexpression in PCNSLs and patient survival may be due to either this unique infiltration style of PCNLs or another role of COX-2 and VEGF in addition to neoangiogenesis in PCNSLs.

Although the mechanisms remain unclear, a recent in vitro investigation indicated that overexpression of COX-2 results in dedifferentiation, adhesion to extracellular matrices, and inhibition of programmed cell death in intestinal cells. Li et al found that COX-2 overexpression is closely related to the p53 mutation status in gastric MALT lymphoma (16). They also referred to COX-2 as the ultimate effector in the p53Ras/Raf/MAPK-COX-2 pathway in gastric MALT lymphoma. In the laboratory examination, Wun et al examined the effects of COX-2 inhibition on apotosis in several B lymphoma cell lines and showed apoptotic induction of $>85 \%$ in all cell lines examined by celecoxib (21). In their examination of apoptosis-related proteins, the levels of BCL-2, BCL-XL and Bax were unaffected, but Akt, MCL-1 and phosphorylated SAP-kinase were all decreased after incubation with the selective $\mathrm{COX}$ inhibitor celecoxib. They, therefore, considered that COX-2 activation and activity contribute to the pathogenesis of B-cell lymphomas. Scarce data are available regarding the potential role of COX-2 in PCNSL pathogenesis. However, the findings of over-expression of COX-2 and VEGF in PCNSLs are in line with those observed previously in various types of tumorigenesis.

Epidemiologic studies have demonstrated that chronic use of a non-steroidal antiinflammatory drug (NSAID) significantly reduces the risk of colon cancer (29). Studies in animal models have also shown colon tumor inhibition by several NSAIDs (30). In hematopoietic neoplasms, Potter et al have shown that the non-selective COX inhibitor blunts the generation of plasmacytomas in a mouse pre-clinical model (31).

Clinical trials in patients with familial polyposis suggest that administration of sulindac, an NSAIDs, induces a reduction of polyps (32). NSAID function could involve blockage of COXs and continuous inhibition of eicosanoids, which affect cell proliferation, cancer growth and immune responsiveness. Interestingly, Reddy et al have assessed the chemopreventive properties of SC-58635, a selective COX-2 inhibitor using azoxymethane-induced colonic aberrant crypt foci (ACF), in rats as a measure of efficiency (7). They found that SC-58635 significantly suppresses colonic ACF formation and multiplicity, and hypothesized that a selective COX-2 inhibitor possesses chemopreventive activity against colon carcinogenesis. Altorki et al have reported that celecoxib, a selective COX-2 inhibitor, enhances the response to preoperative paclitaxel and carboplatin in early-stage non-small cell lung cancer in clinical trials (15). Phipps et al have indicated that celecoxib might have potential therapeutic benefits in the treatment of patients with B-cell lymphomas in combination with other agents (24). These observations, together with the observed overexpression of COX-2 and VEGF staining in PCNSLs in the present study could possibly lead to a future therapeutic trial of PCNSLs with selective COX-2 inhibitor therapy.

\section{Acknowledgements}

We thank Mr. Fumihiro Mutoh (Department of Pathology and Biodefense, Saga University, Faculty of Medicine) for his technical assistance.

\section{References}

1. De Angelis LM, Seiferheld W, Schold SC, Fisher B and Schltz CJ: Combination chemotherapy and radiotherapy for primary central nervous system lymphoma: radiation therapy oncology group study 93-10. J Clin Oncol 20: 4643-4648, 2002.

2. Sugita Y, Tokunaga O, Nakashima A and Shigemori M: SHP-1 expression in primary central nervous system B-cell lymphomas in immunocompetent patients reflects maturation stage of normal B cell counterparts. Pathol Int 54: 659-666, 2004. 
3. Chang C-C, Kampalath B, Schultz C, Bunyi-Teopengco E, Logan B, Eshoa C, Dincer A and Perkins SL: Expression of p53, c-Myc or Bcl-6 suggests for a poor prognosis in primary central nervous system diffuse large B-cell lymphoma among immunocompetent individuals. Arch Pathol Lab Med 127: 208-212, 2003.

4. Roser F, Saini M, Meliss R, Ostertag H, Samii M and Bellinzona M: Apoptosis, vascularity and proliferation in primary central nervous system lymphomas: a histopathological study. Surg Neurol 62: 393-399, 2004.

5. Camilleri-Broët $S$, Crinière E, Broët $P$, Delwail V, Mokhtari K, Moreau A, Kujas M, Raphaël M, Iraqi W, Sautès-Fridman C, Colombat P, Hoang-Xuan K and Martin A: An unform activated B-cell-like immunophenotype might explain the poor prognosis of primary central nervous system lymphomas: analysis of 83 cases. Blood 107: 190-196, 2006.

6. Paulus W, Jellinger K, Morgello S and Deckert-Schluter M: Malignant lymphomas. In: Pathology and Genetics of Tumours of the Nervous System. Kleihues P and Cavenee WK (eds). International Agency for Research on Cancer Press, Lyon, pp198-203, 2000.

7. Reddy BS, Rao CV and Seibert K: Evaluation of cyclooxygenase-2 inhibitor for potential chemopreventive properties in colon carcinogenesis. Cancer Res 56: 4566-4569, 1996.

8. Hida T, Yatabe Y, Achiwa H, Muramatsu H, Kozaki K, Nakamura S, Ogawa M, Mitsudomi T, Sugura T and Takahashi T: Increased expression of cyclooxygenase- 2 occurs frequently in human lung cancers, specifically in adenocarcinomas. Cancer Res 58: 4566-4569, 1998.

9. Zimmermann KC, Sarbia M, Weber A-A, Borchard F, Gabbert HE and Schrör K: Cyclooxygenase-2 expression in human esophageal carcinoma. Cancer Res 59: 198-204, 1999.

10. Sung JJY, Leung WK, Go MYY, To KF, Cheng ASL, Ng EKW and Chan FKL: Cyclooxygenease-2 expression in Helicobacter pylori-associated premalignant and malignant gastric lesions. Am J Pathol 157: 729-735, 2000.

11. Bing RJ, Miyataka M, Rich KA, Hanson N, Wang X, Slosser HD and Shi S-R: Nitric oxide, prostanoids, cyclooxygenase and angiogenesis in colon and breast cancer. Clin Cancer Res 7: 3385-3392, 2001.

12. Shono T, Tofilon PJ, Brunner JM, Owolabi O and Lang F: Cyclooxygenease-2 expression in human gliomas: prognostic significance and molecular correlations. Cancer Res 61: 4375$4381,2001$.

13. Fujiwaki R, Iida K, Kansaki H, Ozaki T, Hata K and Miyazaki K: Cyclooxygenease- 2 expression in endometrial cancer: correlation with microvessel count and expression of vascular endothelial growth factor and thymidine phosphorylase. Hum Pathol 33: 213-219, 2002.

14. Gallo O, Masini E, Bianchi B, Bruschini L, Paglierani M and Franchi A: Prognostic significance of cyclooxygenease-2 pathway and angiogenesis in head and neck squamous cell carcinoma. Hum Pathol 33: 708-714, 2002.

15. Altorki NK, Keresztes RS, Port LJ, Libby DM, Korst RJ, Flieder DB, Ferrara CA, Yankelevitz DF, Subbaramaiah K, Pasmantier MW and Dannenberg AJ: Celecoxib, a selective cyclo-oxygenase-2 inhibitor, enhances the response to preoperative paclitaxel and carboplatin in early-stage non-smallcell lung cancer. J Clin Oncol 21: 2645-2650, 2003.

16. Li H-L, Sun B-Z and Ma F-C: Expression of COX-2, iNOS, p53 and Ki-67 in gastric mucosa-associated lymphoid tissue lymphoma. World J Gastroenterol 10: 1862-1866, 2004.
17. Hazar B, Ergin M, Seyrek E, Erdogan S, Tuncer I and Hakverdi S: Cyclooxygenease-2 (COX-2) expression in lymphomas. Leuk Lymphoma 45: 1395-1399, 2004.

18. Ichinoue M, Mikami T, Shiraishi H and Okayasu I: High microvascular density is correlated with high VEGF, iNOS and COX-2 expression in penetrating growth-type early gastric carcinomas. Histopathology 45: 612-618, 2004.

19. Hara A and Okayasu I: Cyclooxygenease-2 and inducible nitric oxide synthase expression in human astrocytic gliomas: correlation with angiogenesis and prognostic significance. Acta Neuropathol 108: 43-48, 2004.

20. Hemmerlein B, Galuschka L, Putzer N, Zischkau S and Heuser M: Comparative analysis of COX-2, vascular endothelial growth factor and microvessel density in human renal cell carcinomas. Histopathology 45: 603-611, 2004.

21. Wun T, McKnight $\mathrm{H}$ and Tuscano JM: Increased cyclooxygenase-2 (COX-2): a potential role in the pathogenesis of lymphoma. Leuk Res 28: 179-190, 2004.

22. Roma AA and Prayson RA: Expression of cyclo-oxygenase- 2 in ependymal tumors. Neuropathology 26: 422-428, 2006.

23. Buccoliero AM, Caldarella A, Gheri CF, Taddei A, Paglierani M, Pepi M, Mennonna P and Taddei GL: Inducible cyclooxygenase (COX-2) in glioblastoma-clinical and immunohistochemical (COX-2-VEGF) correlations. Clin Neuropathol 25: 59-66, 2006.

24. Phipps RP, Ryan E and Bernstein SH: Inhibition of cyclooxygenase-2: a new targeted therapy for B-cell lymphoma? Leuk Res 28: 109-111, 2004.

25. Sugita Y, Takase Y, Mori D, Tokunaga O, Nakashima A and Shigemori M: Endoglin (CD105) is expressed on endothelial cells in the primary central nevous system lymphomas and correlates with survival. J Neurooncol J Neurooncol 82: 249256, 2007.

26. Yamamoto S, Yasui W, Kitadai Y, Yokozaki H, Haruma K, Kajiyama $G$ and Tahara E: Expression of vascular endothelial growth factor in human gastric carcinomas. Pathol Int 48: 499-506, 1998.

27. Salven P, Orpana A, Teerenhovi L and Joensuu H: Simultaneous elevation in the serum concentrations of the angiogenic growth factors VEGF and bFGF is an independent predictor of poor prognosis in non-Hodgkin lymphoma: a single-institution study of 200 patients. Blood 96: 3712-3718, 2000.

28. Hazar B, Paydas S, Zorldemir S, Sahin B and Tuncer I: Prognostic significance of microvessel density and vascular endothelial growth factor (VEGF) expression in non-Hodgkin's lymphma. Leuk Lymphoma 44: 2089-2093, 2003.

29. Thun MJ, Namboodiri MM and Heath CW Jr: Aspirin use and reduced risk of fatal colon cancer. N Engl J Med 325: 1593-1596, 1991.

30. Jacoby RF, Seibert K, Cole CE, Kelloff G and Lubet RA: The cyclooxygenase- 2 inhibitor celecoxib is a potent preventive and therapeutic agent in the min mouse model of adenomatous polyposis. Cancer Res 60: 5040-5044, 2000.

31. Potter M: Indomethacin inhibition of pristane plasmacytomagenesis in genetically susceptible in bred mice. Adv Exp Med Biol 469: 151-156, 1999.

32. Waddell WR, Gasner G, Cerise EJ and Loughry RW: Sulindac for polyposis of the colon. Am J Surg 157: 175-179, 1989. 